\title{
Bowel Ultrasound: A Non-invasive, Easy to Use Method to Predict the Need to Intensify Therapy in Inflammatory Bowel Disease Patients
}

\author{
Anda Les ${ }^{1,2,3}$, Razvan Iacob ${ }^{1,2,3}$, Roxana Saizu ${ }^{1}$, Bogdan Cotruta ${ }^{1,2}$, Adrian Ionut Saizu ${ }^{1}$, Speranta Iacob ${ }^{1,2,3}$, Liliana \\ Gheorghe $^{1,2,3}$, Cristian Gheorghe $e^{1,2,3}$
}

1) Digestive Diseases and Liver Transplantation Center, Fundeni Clinical Institute, Bucharest;

2) Center of Excellence in Translational Medicine, Fundeni Clinical Institute, Bucharest;

3) Carol Davila University of Medicine and Pharmacy, Bucharest, Romania

Address for correspondence: Razvan Iacob MD PhD, Digestive Diseases and Liver Transplantation Center, Fundeni Clinical Institute, 258 Fundeni Str. 022328 Bucharest, Romania raziacob@gmail.com
Received: 07.05.2021 Accepted: 18.09.2021

\section{ABSTRACT}

Background \& Aims: Bowel ultrasound (BU) is a non-invasive, inexpensive, widely available tool, valuable for inflammatory bowel disease (IBD) assessment. The aim of the present study was to investigate the clinical utility of BU to predict the need to intensify therapy in IBD patients.

Methods: One hundred seventeen IBD patients ( 89 Crohn's disease, and 28 ulcerative colitis) diagnosis established at least 6 months before enrolment, undergoing maintenance therapy were prospectively included in the study. Bowel ultrasound investigated the following parameters: the bowel wall thickness (BWT), loss of wall stratification, the presence of the bowel wall Doppler signal, the visible lymph nodes, the mucosal hyperechoic spots, and the irregular external bowel wall. The patients were followed-up for 6 months, registering the need to escalate the treatment regimen. Subgroup analyses were conducted for patients requiring immediate treatment intensification (37 subjects), due to active disease at baseline and patients with subsequent treatment intensification, in the 6 months follow-up period (21 cases) in comparison to patients that required no therapeutic optimization (59 cases).

Results: During the follow-up, $49.6 \%$ of patients needed treatment escalation. All the investigated BU variables were significantly associated with the main outcome. In the multivariate analysis, the mean BWT $(\mathrm{p}<0.0001)$, and the presence of the bowel wall Doppler signal $(\mathrm{p}=0.007)$ were independent predictors of the main outcome. For the subgroup analyses: mean BWT $(\mathrm{p}=0.0001)$ and the presence of the bowel wall Doppler signal $(\mathrm{p}=0.01)$ were independent predictors for immediate treatment intensification (active disease at baseline) and mean BWT ( $\mathrm{p}=0.0003)$ and the lack of bowel wall stratification $(\mathrm{p}=0.05)$ were independent predictors for the need of subsequent therapeutic optimization. Logistic regression prediction models and prediction scores (BU score) had the best AUROC values ( $>0.91)$ when compared to traditional biomarkers of active inflammation, such as $\mathrm{C}$ reactive protein or fecal calprotectin.

Conclusion: Bowel ultrasound could be used as a non-invasive, easy to use diagnostic tool to predict the need to intensify therapy in patients with IBD.

Key words: inflammatory bowel diseases - ulcerative colitis - Crohn's disease - bowel ultrasound - treatment escalation.

Abbreviations: 5-ASA: 5-aminosalicylic acid; BU: bowel ultrasound; BWT: bowel wall thickness; CD: Crohn's disease; CEUS: contrast-enhanced ultrasound; CRP: C reactive protein; CT: computed tomography; FC: fecal calprotectin; IBD: inflammatory bowel disease; MRI: magnetic resonance imaging; SLIC: Sonographic Lesion Index for Crohn's Disease; TNF: tumor necrosis factor; UC: ulcerative colitis.

\section{INTRODUCTION}

Inflammatory bowel diseases (IBD) [Crohn's Disease (CD) and ulcerative colitis (UC)], are chronic disorders of the digestive tract evolving with alternating episodes of inflammatory flares and remission. During the disease course, multiple endoscopic, biological, and imaging assessments are required to evaluate the disease activity $[1,2]$. Endoscopy, however, has several limitations, as it is invasive, time consuming, has a poor acceptability by the patient [3] and is unable to detect lesions beyond the bowel mucosa. Thus, other imaging techniques are used as complementary diagnostic tools.

Recent guidelines recommend bowel ultrasound (BU) as a complementary imaging technique, together with other cross-sectional imaging modalities, to assess transmural and extraintestinal lesions [4]. Bowel ultrasound has similar 
sensitivity and specificity to other imaging methods such as computed tomography $(\mathrm{CT})$ or magnetic resonance imaging (MRI), for bowel lesions assessment [5]. Significant limitations of the latter consist of the use of ionizing radiation and the high costs, which represent drawbacks for repeated use in IBD monitoring. Alternatively, BU is a safe, rapid, inexpensive, noninvasive technique, easily accepted by patients. It is useful for the IBD diagnosis, for the detection of mural and extramural complications such as stenoses and fistulas, and for disease activity follow-up $[3,6]$. In a recent study, Allocca et al. [7] showed that treatment tailoring based on BU findings, was similar to those based on clinical, biological, endoscopic, or other imaging tools, making ultrasonography a valuable alternative to more expensive imaging methods for IBD patients monitoring.

Calprotectin is a protein found in neutrophils and its presence in the feces reflects neutrophils migration in the digestive tract, due to an active inflammatory process [8]. Its resistance to intestinal secretions and bacterial degradation as well as stability and uniformity in the stool, makes this protein a suitable marker for detecting mucosal inflammation [9]. Fecal calprotectin (FC) has a high sensitivity for detection of IBD lesions ( $87 \%$ ) but a low specificity (67\%), increased levels being detected in infections, diverticulitis, or colorectal cancer $[8,10]$. The utility of this marker, along with other serological inflammatory markers such as $\mathrm{C}$ reactive protein (CRP), in IBD, consists in its diagnostic use, in the assessment of severity of active inflammation, monitoring of the mucosal healing process and in the prediction of disease relapse [11-13].

The aim of our study was to assess, in clinical practice, the ability of BU to predict the need to intensify the therapeutic regimen in patients with IBD, in comparison to traditional disease activity markers, such as CRP and FC.

\section{METHODS}

One hundred and seventeen patients with an established IBD diagnosis addressed to the Digestive Diseases and Liver Transplantation Center, Fundeni Clinical Institute, between January 2018-January 2020, undergoing maintenance therapy, were prospectively included in the study. There were 89 patients with CD and 28 patients with UC enrolled in our study, after informed consent. Only patients undergoing IBD treatment, with a definite diagnosis of IBD established for at least 6 months before enrolment according to current guidelines were included. Patients with a previous history of bowel surgery, younger than 18 years of age, with associated acute infectious colitis or solely proctitis were excluded.

For eligible patients, significant demographic and clinical data were registered. Disease activity was assessed at baseline according to international accepted scoring systems (CDAI and Mayo scores). Before endoscopic evaluation patients were investigated by bowel ultrasound using Arietta 70 Ultrasound Equipment (Hitachi Medical Corporation, Japan). Ultrasound examination was performed after at least 4 hours of fasting, without administration of pegylated solutions or water. Fecal calprotectin and CRP were also quantified at baseline. The investigators were informed about the patient's IBD phenotype but were blinded to biological data at the time of the ultrasonographic assessment. The subjects were subsequently prospectively followed, registering the need to escalate the therapeutic regimen during a subsequent 6-month time frame, according to current guidelines, based on the decision of the attending physician: need for a new corticosteroid course followed by thiopurines or biologicals, biological treatment intensification or biological switch. Patients presenting a disease flare at baseline underwent immediate treatment intensification, otherwise therapeutic intensification during the subsequent 6 months of follow-up was registered (immediate vs subsequent intensification). If patients were under 5-aminosalicylic acid (5-ASA) maintenance therapy, in case of active disease, treatment escalation included a new steroid course followed by thiopurines or initiation of biologicals. For patients undergoing thiopurine maintenance therapy, treatment escalation consisted of biological initiation. For patients undergoing biological therapy, treatment escalation was considered increased dosing, decreasing treatment interval or biological switch.

There were no patients with CMV colitis/reactivation or Clostridium difficile colitis registered in our study group during follow-up.

The ultrasonographic assessment was performed with the patient in supine position. Initial examination was conducted with a $3-5 \mathrm{MHz}$ convex transducer followed by a $5-12 \mathrm{MHz}$ micro-convex transducer. After the general bowel ultrasound assessment, the investigation was focused on pathological segments. The following BU features were investigated: bowel wall thickness (BWT), loss of wall stratification, presence of bowel wall Doppler signal, the presence of hyperechoic spots inside the bowel wall, the irregularity of the external layer of the wall, the presence of creeping fat (mesenteric hypertrophy), the presence of visible lymph nodes adjacent to a pathological bowel loop. There were 3 sonographic measurements of BWT performed, registering in the database the mean value. When more than one segment was involved, the most severe alteration was assessed and recorded in the database. Furthermore, Doppler signal was adapted to low velocity, to assess small vessels blood flow. Complications such as stenosis, fistulas and abscesses were registered, if encountered. Stenoses were confirmed when a thickened bowel loop was identified among with significant distension $(\geq 25 \mathrm{~mm})$ and, whether Doppler signal or stratification were present, classified into fibrous or inflammatory. Fistulas were seen as communicating hypoechoic areas between two bowel segments or other structures and abscesses were confirmed when hypoechoic areas with irregular wall and hyperechoic spots suggesting the presence of air was seen.

The Institutional Ethics Committee of our Institution approved the study protocol and all patients gave written informed consent prior to the inclusion in the study.

For categorical variables univariate analysis was conducted by chi-square test or Fisher exact, as appropriate. Quantitative variableswere compared between study groups using the Student's $t$ test or the Mann Whitney U test. Significant variables according to the univariate analysis were included in a multivariate analysis by logistic regression, to identify independent predictors of the outcome. The clinical utility for prediction of the outcome was assessed by the means of 
the ROC curve. A two tailed p value $<0.05$ was considered for statistical significance. Statistical analysis was conducted using NCSS v9.0.7 (LLC).

\section{RESULTS}

Patient characteristics are depicted in Table I. There was a significantly longer disease duration for patients with CD in our study group. A significantly higher proportion of patients were under anti-tumor necrosis factor (anti-TNF) maintenance therapy in the CD group, whereas a higher percentage of cases were under 5-amino-salicylates (5-ASA) treatment in the UC group, as expected. The other clinical variables were not significantly different between CD and UC patients.

Main BU features are presented in Table II. There was a higher mean BWT in patients with CD. Loss of bowel wall stratification was encountered in $43.6 \%$ of cases in our study group, in a significantly higher proportion of cases diagnosed with CD. Bowel wall Doppler signal was detected in $62.4 \%$ of investigated cases, mesenteric hypertrophy in $59.8 \%$, mucosal hyperechoic spots in $28.2 \%$ and irregular external wall in $32.8 \%$ of cases, with no significant difference between CD and UC cases. Visible lymph nodes could be identified on bowel ultrasound in $45.3 \%$ of subjects, with no statistically significant difference between IBD phenotypes. Significant bowel ultrasound features are exemplified in Fig. 1.
During follow-up, the need for treatment escalation (study outcome) was registered in $49.6 \%$ of cases, in our study group. Thirty-seven subjects $(31.7 \%)$ required immediate treatment intensification due to disease flare at baseline, whereas 21 cases (17.9\%) underwent subsequent treatment intensification during the 6 months follow-up. Mean CRP value in the study subgroup that required immediate treatment intensification was $67.5 \mathrm{mg} / \mathrm{dl}$ vs $7.7 \mathrm{mg} / \mathrm{dl}$ in the subgroup of patients that required no treatment intensification $(p<0.0001)$. In the subgroup of patients with subsequent treatment intensification mean CRP value was $5.4 \mathrm{mg} / \mathrm{dl}(\mathrm{p}=0.29$ vs no treatment intensification), suggesting a low predictive value for the need of subsequent therapeutic step-up for CRP, outside an active disease flare. Mean FC value in the study subgroup that required immediate treatment intensification was 1092 $\mu \mathrm{g} / \mathrm{g}$ vs $271.5 \mu \mathrm{g} / \mathrm{g}$, in the subgroup of patients that required no treatment intensification $(\mathrm{p}<0.0001)$. In the subgroup of patients with subsequent treatment intensification mean FC value was $612.7 \mu \mathrm{g} / \mathrm{g}$ ( $\mathrm{p}=0.01$ vs no treatment intensification), supporting a better predictive value of FC for the need of subsequent therapeutic optimization.

In a univariate analysis, all the studied bowel ultrasound features but not the disease phenotype were significantly associated with the outcome (Table III).

In the multivariate analysis, only mean BWT and the presence of bowel wall Doppler signal were independent

Table I. Patient's characteristics according to IBD phenotype

\begin{tabular}{|c|c|c|c|}
\hline & Crohn's Disease & Ulcerative colitis & $\mathbf{p}$ \\
\hline Patients, n (\%) & $89(76 \%)$ & $28(23.9 \%)$ & N/A \\
\hline Age (years, mean $\pm \mathrm{SD}$ ) & $37.4 \pm 12.6$ & $38.1 \pm 15.2$ & 0.94 \\
\hline Male, n (\%) & $44(49.4 \%)$ & $12(42.9 \%)$ & 0.69 \\
\hline Body mass index $\left(\mathrm{kg} / \mathrm{m}^{2}\right.$, mean $\left.\pm \mathrm{SD}\right)$ & $22.8 \pm 3.8$ & $24 \pm 4.9$ & 0.36 \\
\hline \multicolumn{4}{|l|}{ Montreal phenotypic classification } \\
\hline Age at diagnosis (A1/A2/A3) & $5 / 67 / 17(5.6 \% / 75.3 \% / 19.1 \%)$ & N/A & N/A \\
\hline Disease location (L1/L2/L3/L4) & $30 / 30 / 29 / 0(33.7 \% / 33.7 \% / 32.6 \% / 0 \%)$ & N/A & N/A \\
\hline Disease behaviour (B1/B2/B3/p) & $38 / 38 / 13 / 6(42.7 \% / 42.7 \% / 14.6 \% / 6.8 \%)$ & $\mathrm{N} / \mathrm{A}$ & N/A \\
\hline Disease extension (E1/E2/E3) & N/A & $0 / 11 / 17(0 \% / 39.3 \% / 60.7 \%)$ & N/A \\
\hline \multicolumn{4}{|l|}{ Disease severity } \\
\hline CDAI & $188.9 \pm 111.4(10-448)$ & N/A & N/A \\
\hline Mayo & $\mathrm{N} / \mathrm{A}$ & $6.7 \pm 3.9(0-11)$ & N/A \\
\hline Time from diagnosis (years, mean \pm SD) & $6.9 \pm 6$ & $4.2 \pm 3.7$ & 0.03 \\
\hline Fecal calprotectin $(\mu \mathrm{g} / \mathrm{g}$, mean $\pm \mathrm{SD})$ & $617.6 \pm 614.2$ & $776.9 \pm 932$ & 0.98 \\
\hline $\mathrm{CRP}(\mathrm{mg} / \mathrm{dl}$, mean $\pm \mathrm{SD})$ & $29.6 \pm 59.2$ & $15.6 \pm 24.7$ & 0.49 \\
\hline Serum albumin ( $\mathrm{g} / \mathrm{dl}$, mean $\pm \mathrm{SD})$ & $3.9 \pm 0.7$ & $3.8 \pm 0.9$ & 0.98 \\
\hline Hemoglobin $(\mathrm{g} / \mathrm{dl}$, mean $\pm \mathrm{SD})$ & $12.5 \pm 2.2$ & $13 \pm 2.4$ & 0.31 \\
\hline Baseline maintenance therapy & & & 0.02 \\
\hline 5-ASA & $23(25.8 \%)$ & $15(53.6 \%)$ & \\
\hline Budesonide & $12(13.5 \%)$ & $0(0 \%)$ & \\
\hline AZA & $9(10.1)$ & $3(10.7 \%)$ & \\
\hline Anti-TNF & $45(50.6 \%)$ & $10(35.7 \%)$ & \\
\hline
\end{tabular}

A: age; A1 <16 years; A2 between 17 and 40 years; A3 > 40 years; L: location: L1: ileal; L2: colonic; L3: ileocolonic; L4: isolated upper disease; B: behaviour; B1: non-stricturing, non-penetrating; B2: stricturing; B3: penetrating; p: perianal disease; E1: ulcerative proctitis; E2: left sided ulcerative colitis; E3: extensive ulcerative colitis; CDAI: Crohn's disease activity index; CRP: C reactive protein; 5-ASA: 5-aminosalicylic acid; AZA: azathioprine; TNF: tumor necrosis factor; N/A: not applicable. 
Table II. Bowel ultrasound findings, according to IBD phenotype

\begin{tabular}{lccc}
\hline & Crohn's Disease & $\begin{array}{c}\text { Ulcerative } \\
\text { Colitis }\end{array}$ & $\mathrm{p}$ \\
\hline Mean BWT (mm, mean \pm SD) & $6 \pm 2.3$ & $4.9 \pm 1.9$ & 0.01 \\
Loss of wall stratification, n (\%) & $47(52.8)$ & $4(14.3)$ & 0.0007 \\
Bowel wall Doppler signal, n (\%) & $58(65.1)$ & $15(53.6)$ & 0.37 \\
Mesenteric hypertrophy, n (\%) & $58(65.1)$ & $12(42.9)$ & 0.06 \\
Visible lymph nodes, n (\%) & $42(47.2)$ & $11(39.3)$ & 0.6 \\
Mucosal hyperechoic spots, n (\%) & $27(30.3)$ & $6(21.4)$ & 0.5 \\
Irregular external wall, n (\%) & $32(36.4)$ & $6(21.4)$ & 0.21 \\
\hline
\end{tabular}

BWT: bowel wall thickness; SD: standard deviation.

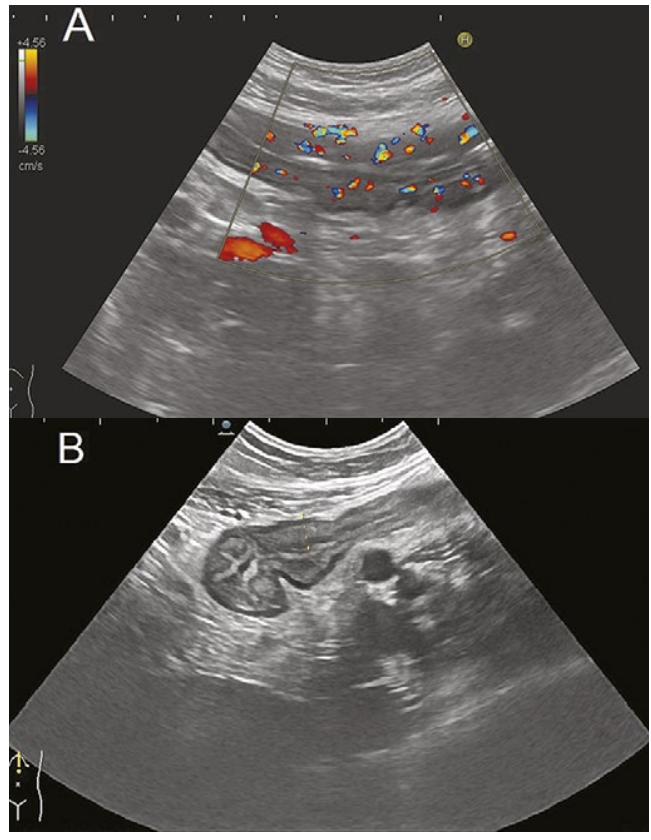

Fig. 1. Bowel ultrasound features: A) Increased color Doppler signal of the bowel wall signifying active inflammation; B) increased bowel wall thickness.

predictors of the main outcome (Table IV). Subgroup multivariate analyses were conducted to investigate $\mathrm{BU}$ variables as independent predictors for immediate treatment optimization or for subsequent treatment optimization, during the 6-month follow-up. Based on the independent predictors, logistic regression models were generated and BU Scores to evaluate the need of IBD treatment intensification were calculated (Table IV). For the outcome "Need to intensify treatment in 6 months": Bowel Ultrasound Score (BU Score) $=1 /(1+\operatorname{Exp}(-\mathrm{XB}))$ where $\mathrm{XB}=0.88^{*}$ [bowel wall thickness $(\mathrm{mm})]+2.02^{*}$ (Doppler $\left.=1\right)-6.67$.

The AUROC of the BU score, as a predictor for the need to intensify treatment in 6 months, in patients with IBD was 0.91 , indicating a good clinical utility. A BU score value $>0.45$ has a $96.6 \%$ sensitivity, $83 \%$ specificity, $84.8 \%$ positive predictive value and $96.1 \%$ negative predictive value for prediction of the study outcome. For the same outcome, AUROC for CRP was 0.81 ( $\mathrm{p}=0.05$ vs BU score) whereas for fecal calprotectin it was 0.85 ( $\mathrm{p}=0.2$ vs BU score) (Fig. $2 \mathrm{~A}$ ). The cut-off value $>0.45$ of the BU score successfully predicted the need to intensify treatment in 6 months in 56 out of 58 patients. However, the same cut-off value also indicated a need for therapeutic intensification in 11 cases that did not actually undergo a treatment intensification during the 6 months follow-up, according to current disease activity criteria. Out of these 11 IBD cases, 10 were diagnosed with $\mathrm{CD}$, with a mean CDAI at baseline of 204.

For the outcome "Immediate treatment intensification, due to active disease": $\mathrm{BU}$ score $=1 /(1+\operatorname{Exp}(-\mathrm{XB}))$ where $\mathrm{XB}$ $=0.75^{*}[$ bowel wall thickness $(\mathrm{mm})]+3.5^{*}($ Doppler $=1)-7.31$.

Table III. Bowel ultrasound findings according to study outcome

\begin{tabular}{lcccc}
\hline & \multicolumn{4}{c}{ Need to intensify therapy } \\
\hline & $\begin{array}{c}\text { Immediate } \\
\text { intensification }(\mathrm{n}=37)\end{array}$ & $\begin{array}{c}\text { Subsequent } \\
\text { intensification }(\mathrm{n}=21)\end{array}$ & $\begin{array}{c}\text { No intensification } \\
(\mathrm{n}=59)\end{array}$ & $\mathrm{p}$ \\
\hline Mean BWT (mm, mean \pm SD) & $7.6 \pm 1.7^{*}$ & $6.9 \pm 1.3^{*}$ & $4.2 \pm 1.7$ & $<0.0001^{*}$ \\
Loss of wall stratification, $\mathrm{n}(\%)$ & $24(64.9)$ & $15(71.43 \%)$ & $12(20.3 \%)$ & $<0.0001$ \\
Bowel wall Doppler signal, $\mathrm{n}(\%)$ & $36(97.3)$ & $18(85.7 \%)$ & $19(32.2 \%)$ & $<0.0001$ \\
Mesenteric hypertrophy, $\mathrm{n}(\%)$ & $34(91.9)$ & $18(85.7 \%)$ & $18(30.5 \%)$ & $<0.0001$ \\
Visible lymph nodes, $\mathrm{n}(\%)$ & $24(64.9)$ & $11(52.4 \%)$ & $18(30.5 \%)$ & 0.003 \\
Mucosal hyperechoic spots, n (\%) & $19(51.3)$ & $7(33.3 \%)$ & $7(11.9 \%)$ & 0.0001 \\
Irregular external wall, $\mathrm{n}(\%)$ & $23(62.1)$ & $9(42.9 \%)$ & $7(11.9 \%)$ & $<0.0001$ \\
Disease phenotype Crohn's disease, $\mathrm{n}(\%)$ & $26(70.3)$ & $19(90.5 \%)$ & $44(74.6 \%)$ & 0.2 \\
\hline
\end{tabular}

Immediate intensification due to disease flare at baseline. Subsequent intensification during the 6 months follow-up. No treatment intensification during follow-up. Mean bowel wall thickness (BWT) was compared between study groups: "Immediate" and "Subsequent intensification" vs. "No intensification" * (Mann-Whitney U Test). Categorical variables were compared using chi square test. SD: standard deviation. 
Table IV. Bowel ultrasound parameters as independent predictors for treatment intensification, multivariate analysis.

\begin{tabular}{|c|c|c|c|c|c|}
\hline Variable & Coefficient & Std. Error & Odds ratio & $95 \% \mathrm{CI}$ & $\mathbf{p}$ \\
\hline Mean BWT (mm) & 0.9 & 0.25 & 2.4 & $1.5-4$ & 0.0003 \\
\hline Presence of wall stratification & -0.4 & 0.6 & 0.6 & $0.2-2.3$ & 0.5 \\
\hline Bowel wall Doppler signal & 2 & 0.9 & 7.3 & $1.3-41.3$ & 0.02 \\
\hline Mesenteric hypertrophy & -0.009 & 0.9 & 1 & $0.2-6$ & 0.9 \\
\hline Visible lymph nodes & -0.3 & 0.6 & 0.7 & $0.2-2.6$ & 0.6 \\
\hline Mucosal hyperechoic spots & -0.4 & 0.7 & 0.7 & $0.2-2.7$ & 0.6 \\
\hline Irregular external wall & 0.2 & 0.7 & 1.2 & $0.3-4.7$ & 0.8 \\
\hline \multicolumn{6}{|c|}{ Predictive model for the need to intensify therapy in 6 months } \\
\hline Constant & -6.6 & 1.3 & - & - & $<0.0001$ \\
\hline Mean BWT (mm) & 0.9 & 0.2 & 2.4 & $1.6-3.6$ & $<0.0001$ \\
\hline Bowel wall Doppler signal & 2 & 0.7 & 7.6 & $1.7-33$ & 0.007 \\
\hline \multicolumn{6}{|c|}{ Predictive model for immediate treatment intensification, due to active disease } \\
\hline Constant & -7.31 & 2.0 & - & - & 0.0002 \\
\hline Mean BWT (mm) & 0.75 & 0.2 & 2.1 & $1.4-3.1$ & 0.0001 \\
\hline Bowel wall Doppler signal & 3.5 & 1.4 & 32.1 & $1.9-529.2$ & 0.01 \\
\hline \multicolumn{6}{|c|}{ Predictive model for the need of subsequent treatment intensification during a 6 months time frame } \\
\hline Constant & -3.82 & 1.5 & - & - & 0.008 \\
\hline Mean BWT (mm) & 0.8 & 0.2 & 2.2 & 1.4-3.5 & 0.0003 \\
\hline Presence of wall stratification & -1.31 & 0.7 & 0.3 & $0.01-1.01$ & 0.05 \\
\hline
\end{tabular}

Generation of logistic regression prediction models for the specified outcomes, including the most important predictors. A hierarchical forward variable selection method was used for predictive model generation. BWT: bowel wall thickness; Std: standard; CI: confidence interval.

The AUROC of the BU score for this specific outcome was 0.94, promising a good clinical application. A BU score of $>0.5$ had $100 \%$ sensitivity and $83 \%$ specificity, $78.7 \%$ positive predictive value and $100 \%$ negative predictive value. For this outcome, AUROC for FC and CRP were 0.86 and 0.81 respectively ( $\mathrm{p}=\mathrm{NS}$ in comparison to BU score) (Fig. 2B).

For the outcome "Subsequent treatment intensification during a 6 months' time frame": BU score $=1 /(1+\operatorname{Exp}(-\mathrm{XB}))$ where $\mathrm{XB}=0.8^{\star}[$ bowel wall thickness $(\mathrm{mm})]-1.3^{\star}$ ( Presence of wall stratification $=1)-3.82$. The AUROC of the BU score for this specific outcome was 0.92 . A BU Score of $>0.6$ had $90 \%$ sensitivity and $86.4 \%$ specificity, $63 \%$ positive predictive value and $96 \%$ negative predictive value. For this outcome AUROC for FC and CRP were 0.81 and 0.55 respectively (no significant difference for $\mathrm{FC}$ in comparison to $\mathrm{BU}$ score and $\mathrm{p}=0.0004$ for CRP vs BU Score) (Fig. 2C).

Twenty-seven patients (23.3\%) had intestinal complications (stenoses, intestinal fistulas or abscesses) in our study group. Intestinal complications were encountered in $35 \%$ of patients that required therapeutic step-up vs $11.9 \%$ of patients with no treatment optimization during follow-up $(\mathrm{p}=0.006)$. All abscesses were treated by interventional radiology and followed by treatment intensification.

\section{DISCUSSION}

In the present study, we have shown that BU features can accurately predict the need to escalate therapy in a six months'

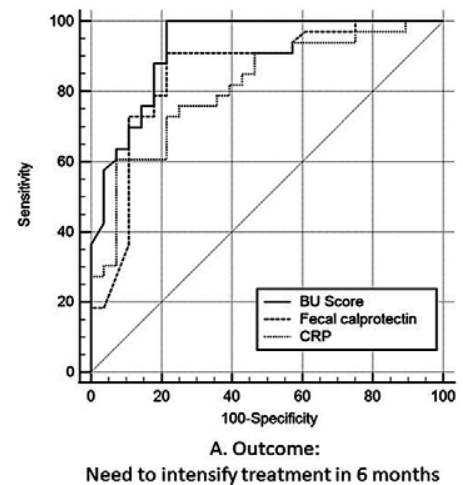

Fig. 2. AUROC for study outcomes (A, B, C) for bowel ultrasound (BU) score, baseline fecal calprotectin (FC) and C reactive protein (CRP).

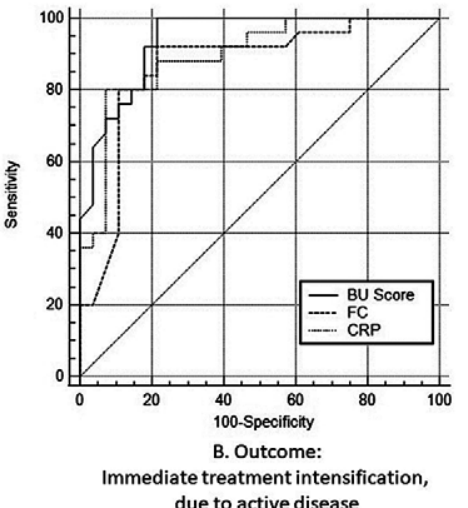

due to active disease

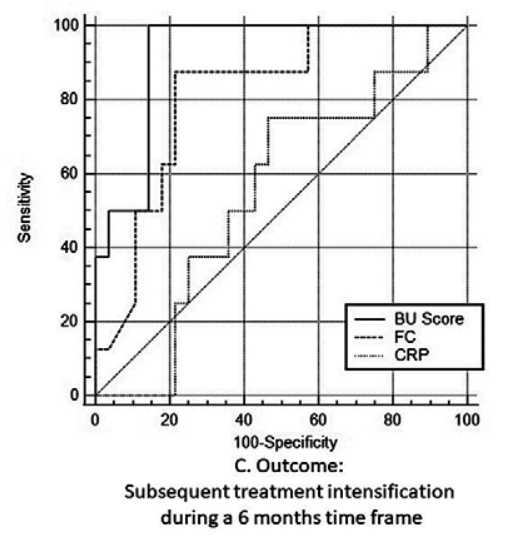

J Gastrointestin Liver Dis, December 2021 Vol. 30 No 4: 462-469 
time frame, in patients with CD and UC. Several easy to generate and reproducible BU variables were assessed. BWT and the presence of intramural Doppler signal were identified as independent predictors of the outcome, by our multivariate analysis.

In $\mathrm{CD}, \mathrm{BU}$ has a similar diagnostic accuracy and similar ability to monitor disease activity compared to other imaging methods (CT, MRI) $[5,14,15]$. Bowel wall thickness is one of the most useful BU variables in IBD assessment and one of the most frequently used parameters in clinical trials [16]. A cut-off value of $3 \mathrm{~mm}$ for BWT has a sensitivity of $89 \%$ and a specificity of $96 \%$ for CD diagnosis [16]. Higher BWT value is a common predictor for disease recurrence after surgery in CD. Furthermore, the lack of decrease in BWT after treatment was correlated with a high risk of surgery [16]. In UC, BWT was correlated with clinical activity, biological markers of inflammation and endoscopic lesions, being an accurate tool to detect active disease and to assess response to treatment [16, 17]. As expected, in our study group there was a significantly higher mean BWT in patients with CD compared to UC. However, in the multivariate analysis and in the prediction models, we included the absolute value of the mean BWT as a covariate, without using a specific cut-off, so that the models are applicable to both CD and UC patients.

Novak et al. [18] demonstrated that BU is useful for clinical decision making, in a prospective study including 49 CD patients. Two IBD specialists reviewed the cases and developed a management plan, based on the clinical setting. A separate physician subsequently performed BU measuring BWT, color Doppler flow, presence of lymph nodes, hypertrophic mesentery, integrating these BU data into an overall activity score. After US examination, the two IBD physicians independently reported their clinical management plan. Previous clinical decisions of the two independent IBD specialists were changed after ultrasound assessment in 30/49 (60\%) and 28/48 (58\%) of cases, respectively. A significant proportion of the patients (59\%) were asymptomatic, with an Harvey-Bradshaw index of 3 or less $(n=29)$. However, $52 \%$ $(n=15)$ of cases in the study group had active disease by US assessment, leading to a change of clinical management [18].

Calabrese et al. [19] have developed the Sonographic Lesion Index for Crohn's Disease (SLIC) that quantifies small bowel damage in $\mathrm{CD}$, by contrast ultrasonography using $375 \mathrm{ml}$ of polietilenglycol. Following variables were assessed, to generate 5 different grading classes to describe small bowel damage and extent: BWT, the lumen diameter, small bowel dilation, fistulas, creeping fat, lymph nodes and abscesses. The method was able to identify patients with higher scores that were at risk for surgery during one year of follow up [19]. Zorzi et al. [20] have evaluated 29 CD patients after anti-TNF treatment initiation, using the SLIC score. A significant improvement of SLIC and SLIC sub-scores after induction of remission with anti-TNFs was observed. A significantly decrease of SLIC score and subscores was identified only in clinical responders, proving that BU is useful for treatment follow-up [20]. In another study, the group of Antonio Rispo from Naples evaluated treatment response using $\mathrm{BU}$ and ileocolonoscopy and found that there was a good concordance between the two techniques $(\mathrm{k}=0.63)$ [21].
In our study, clinical decision for treatment escalation was made by the attending physician based on clinical, biological, and endoscopic features suggesting active disease, according to current guidelines $[1,2]$. BU examination was conducted by an independent investigator and findings were correlated to study outcomes. In the univariate analysis there was no correlation between disease phenotype and the study outcomes. Our findings indicate that the use of BU features could support the need of treatment intensification at baseline even better than traditional disease activity biomarkers (CRP and FC). Out of the studied BU variables, mean BWT and bowel wall Doppler signal are equally important for predicting the need of immediate treatment intensification due to active disease or the need for treatment step-up in 6 months. Excluding clinically active disease at baseline, for subsequent treatment intensification during the 6 months follow-up period, BWT and the absence of bowel wall stratification are the best predictors of the outcome. The lack of bowel wall structure could be an early indicator of subclinical inflammation, emphasizing the need of close follow-up, whereas bowel wall Doppler signal is an indicator of active inflammation and has a limited value in these setting.

Rigazio et al. [22] prospectively evaluated 55 consecutive $\mathrm{CD}$ surgical candidates that had a BU examination one month before surgery. The control group of non-surgical IBD cases comprised 110 subjects. BWT, bowel echo-pattern and complications were recorded. The multivariate analysis included BU variables along with clinical variables (disease location, behavior, age at diagnosis, smoking, previous surgery, duration of the disease, gender, extraintestinal manifestation, Harvey-Bradshaw index). The variables significantly associated with the need for surgery were: bowel wall pattern, thickness, evidence of fistula or abscesses and stenoses. A score based on bowel pattern disruption, BWT $>4.5 \mathrm{~mm}$ and the presence of complications was generated. A score $>3$ had an AUC of 0.902 for IBD surgery, with a sensitivity of $79.6 \%$ and a specificity of $85.7 \%$ [22]. In our study we did not consider the need for surgery as an outcome, as in our study group, during the respective follow-up, no patient was referred to surgery. The clinical utility of our score for this outcome should be investigated in subsequent studies, with longer follow-up.

The Humanitas ultrasound criteria (HUC) were generated by examining 53 UC patients of which 22 had mucosal healing assessed by colonoscopy. BWT higher than $3 \mathrm{~mm}$, color Doppler flow, hypoechoic bowel wall and the presence of visible lymph nodes were correlated to endoscopic activity, whereas BWT and Doppler flow were independent predictors for endoscopic activity. Simultaneously, BWT higher than $3 \mathrm{~mm}$ and fecal calprotectin higher than $101 \mathrm{ug} / \mathrm{g}$ had a $100 \%$ sensitivity to predict endoscopic activity. However, FC was not significant in the multivariate analysis. ROC analysis identified $\mathrm{HUC} \geq 6.3$ as a threshold to discriminate patients with active UC vs. non-active UC, with sensitivity of $71 \%$ and a specificity of 100\% (AUC 0.89) [23]. We also identified BWT and bowel wall Doppler signal as independent predictors for the need to intensify treatment, in both CD and UC cases. Parente et al. [24] enrolled 83 patients with moderate to severe UC requiring high-dose steroids and evaluated the accuracy of a US activity index to assess response to therapy and the risk of subsequent 
relapse. A comparison was made to the traditional clinical and endoscopic scores. Endoscopic severity of UC was graded 0-3 according to the Baron score, and US severity $0-3$, according to the colonic wall thickening and the presence of vascular signal at power Doppler. A high US score of 2 or 3 at 3-month assessment, predicted a severe endoscopic activity at 15 months of follow-up [24]. Novak et al. [25] have generated a BU score to predict CD disease activity in a cohort of 160 cases, that was subsequently validated on an independent cohort of 63 cases. The following BU parameters were analysed: BWT, colour Doppler flow, mesenteric inflammatory fat, lymph nodes and the presence of complications. In concordance with our study, BWT and colour Doppler signal exhibited the strongest predictability of disease activity. The score had an AUROC of 0.866 and 0.836 for predicting disease activity, in the two cohorts, respectively [25].

The International Bowel Ultrasound Group proposed a segmental activity score that predicts disease activity in CD patients. Data was provided by 12 international experts that evaluated $30 \mathrm{CD}$ cases on a visual analogue scale and concluded that BWT, color Doppler signal, presence of mesenteric fat stranding and bowel wall stratification predict overall disease activity. The score demonstrated an intraclass correlation coefficient of 0.97 indicating excellent reliability but should be validated in subsequent studies [26].

Recently, two new sonographic scores have been published. Bots et al. [27] have elaborated an UC score based on BWT, Doppler signal presence, presence of haustration and fat wrapping on 60 patients and each colonic segment, except for the rectum, was analysed in comparison to Mayo and UCEIS scores. Strong correlations were observed between the UC ultrasound score and endoscopic scores ( $\mathrm{rho}=0.83$ and 0.75 respectively, $\mathrm{p}<0.001)$. The score should also be validated by future studies [27]. Saevik et al. [28] developed an US score in a 40 CD patients cohort, that was subsequently validated on 124 patients. The initial CD ultrasonographic score was calculated based on BWT, colour Doppler signal, fatty wrapping, and stratified structure of the bowel wall. As the latter 2 variables did not contribute significantly to the model, only BWT and colour Doppler were integrated in the CD US score. The score strongly correlated with the SES-CD score ( $r h o=0.83, p<0.001)$. In the validation cohort, the score also correlated well with SES-CD, but with slightly lower values ( $r h o=0.78, \mathrm{p}<0.001$ ). ROC curve analysis has indicated an area under the curve of 0.92 for an endoscopic SES-CD score $>2$, and a score cut-off $\geq 1$ yielded a sensitivity of $95.3 \%$ and specificity of $70.3 \%$ for the outcome [28].

It has been shown that contrast-enhanced ultrasound (CEUS) could be used to provide accurate information on the active inflammation enhancing mural and mesenteric blood flow $[29,30]$. In our study, however, we did not usd CEUS to evaluate active inflammation but rather used colour Doppler ultrasound evaluation which does not require the use of a contrast agent and equally provides information on active disease.

In clinical practice, high disease activity translates in the need to intensify therapy, thus, we have selected this outcome in our study. One of the advantages of using BU is that it accurately provides non-invasive transmural morphological assessment. The parameters used to calculate the BU scores are easy to obtain in clinical practice and, by showing significant correlation with study outcomes, we have further confirmed their practical utility. The independence from the clinical phenotype of IBD is a strength of our score. As it could be noted by cited references, several studies have separately documented the utility of the same BU variables for predicting disease activity, both in CD and UC. Our study is the first to use a mixed cohort of IBD phenotypes (both CD and UC), prospectively, consecutively recruited, supporting the clinical setting of our daily practice. It is also noteworthy that our score predicted the need for treatment escalation in a subgroup of 11 cases, mostly CD, with a moderate disease activity CDAI score, that did not undergo an actual treatment change during the 6 months follow-up. In future studies, the integration of the BU scores in clinical decision-making algorithms should be evaluated, as it could potentially improve patient management and long-term disease course.

\section{CONCLUSIONS}

As the "treat to target concept" is the currently accepted treatment paradigm, incorporating BU in IBD patients monitoring provides an easy-to-use and readily available tool to stratify patients in need for treatment escalation.

\section{Conflicts of interest: None to declare.}

Author's contribution: A.L., R.I. designed the study, collected and analyzed data, drafted the manuscript, revised the paper. A.L., R.I., R.S., B.C., A.I.S., S.I, L.G collected and analyzed data, drafted the manuscript, revised the paper. C.G. supervised the study, critically revised the manuscript. All the authors approved the final version of the manuscript.

\section{REFERENCES}

1. Gomollón F, Dignass A, Annese V, et al. 3rd European evidence-based Consensus on the diagnosis and management of Crohn's disease 2016: Part 1: Diagnosis and medical management. J Crohns Colitis 2017;11:325. doi:10.1093/ecco-jcc/jjw168

2. Magro F, Gionchetti P, Eliakim R, et al. Third European evidence-based Consensus on diagnosis and management of ulcerative colitis. Part 1: Definitions, diagnosis, extra-intestinal manifestations, pregnancy, cancer surveillance, surgery, and ileo-anal pouch disorders. J Crohns Colitis 2017;11:649-670. doi: 10.1093/ecco-jcc/jjx008

3. Noiseux I, Veilleux S, Bitton A, et al. Inflammatory bowel disease patient perceptions of diagnostic and monitoring tests and procedures. BMC Gastroenterology 2019;19:30. doi:10.1186/s12876-019-0946-8

4. Maaser C, Sturm A, Vavricka SR, et al. ECCO-ESGAR Guideline for diagnostic assessment in IBD Part 1: Initial diagnosis, monitoring of known IBD, detection of complications. J Crohns Colitis 2019;13:144164. doi:10.1093/ecco-jcc/jjy113

5. Allocca M, Fiorino G, Danese S. Cross-sectional imaging modalities in Crohn's disease. Dig Dis 2013;31:199-201. doi:10.1159/000353692

6. Kucharzik T, Kannengiesser K, Petersen F. The use of ultrasound in inflammatory bowel disease. Ann Gastroenterol 2017;30:135-144. doi:10.20524/aog.2016.0105

7. Allocca M, Fiorino G, Bonifacio C, et al. Comparative accuracy of bowel ultrasound versus magnetic resonance enterography in combination with 
colonoscopy in assessing Crohn's disease and guiding clinical decisionmaking. J Crohns Colitis 2018;12:1280-1287. doi:10.1093/ecco-jcc/jjy093

8. Pathirana WGW, Chubb SP, Gillett MJ, Vasikaran SD. Faecal calprotectin. Clin Biochem Rev 2018;39:77-90.

9. Bjarnason I. The use of fecal calprotectin in inflammatory bowel disease. Gastroenterol Hepatol (N Y) 2017;13:53-56.

10. Mosli MH, Zou G, Garg SK, et al. C-reactive protein, fecal calprotectin and stool lactoferrin for detection of endoscopic activity in symptomatic inflammatory bowel disease patients: a systematic review and metaanalysis. Am J Gastroenterol 2015;110:802-819. doi:10.1038/ajg.2015.120

11. Menees SB, Powell C, Kurlander J, Goel A, Chey WD. A meta-analysis of the utility of C-reactive protein, erythrocyte sedimentation rate, fecal calprotectin, and fecal lactoferrin to exclude inflammatory bowel disease in adults with IBS. Am J Gastroenterol 2015;110:444-454. doi:10.1038/ ajg.2015.6

12. Burri $\mathrm{E}$, Beglinger $\mathrm{C}$. The use of fecal calprotectin as a biomarker in gastrointestinal disease. Expert Rev Gastroenterol Hepatol 2014;8:197210. doi:10.1586/17474124.2014.869476

13. Heida A, Park KT, van Rheenen PF. Clinical Utility of fecal calprotectin monitoring in asymptomatic patients with inflammatory bowel disease: A systematic review and practical guide. Inflamm Bowel Dis 2017;23:894-902. doi:10.1097/MIB.0000000000001082

14. Horsthuis K, Bipat S, Bennink RJ, Stoker J. Inflammatory bowel disease diagnosed with US, MR, Scintigraphy, and CT: Meta-analysis of prospective studies. Radiology 2008;247:64-79. doi:10.1148/ radiol.2471070611

15. Bryant RV, Friedman AB, Wright EK, et al. Gastrointestinal ultrasound in inflammatory bowel disease: an underused resource with potential paradigm-changing application. Gut 2018;67:973-985. doi:10.1136/ gutjnl-2017-315655

16. Nylund K, Maconi G, Hollerweger A, et al. EFSUMB Recommendations and Guidelines for Gastrointestinal Ultrasound - Part 1: Examination Techniques and Normal Findings (Short version). Ultraschall Med 2017;38:273-284. doi:10.1055/s-0042-115410

17. Maconi G, Ardizzone S, Parente F, Bianchi Porro G. Ultrasonography in the evaluation of extension, activity, and follow-up of ulcerative colitis. Scand J Gastroenterol 1999;34:1103-1107. doi:10.1080/003655299750024904

18. Novak K, Tanyingoh D, Petersen F, et al. Clinic-based Point of Care Transabdominal Ultrasound for Monitoring Crohn's Disease: Impact on Clinical Decision Making. J Crohns Colitis 2015;9:795-801. doi:10.1093/ecco-jcc/jjv105

19. Calabrese E, Zorzi F, Zuzzi S, et al. Development of a numerical index quantitating small bowel damage as detected by ultrasonography in Crohn's disease. J Crohns Colitis 2012;6:852-860. doi:10.1016/j. crohns.2012.01.015

20. Zorzi F, Stasi E, Bevivino G, et al. A Sonographic Lesion Index for Crohn's Disease Helps Monitor Changes in Transmural Bowel Damage During Therapy. Clin Gastroenterol Hepatol 2014;12:2071-2077. doi:10.1016/j.cgh.2014.04.036

21. Castiglione F, Testa A, Rea M, et al. Transmural healing evaluated by bowel sonography in patients with Crohn's disease on maintenance treatment with biologics. Inflamm Bowel Dis 2013;19:1928-1934. doi:10.1097/MIB.0b013e31829053ce

22. Rigazio C, Ercole E, Laudi C, et al. Abdominal bowel ultrasound can predict the risk of surgery in Crohn's disease: proposal of an ultrasonographic score. Scand J Gastroenterol 2009;44:585-593. doi:10.1080/00365520802705992

23. Allocca M, Fiorino G, Bonovas S, et al. Accuracy of humanitas ultrasound criteria in assessing disease activity and severity in ulcerative colitis: A prospective study. J Crohns Colitis 2018;12:1385-1391. doi:10.1093/ecco-jcc/jjy107

24. Parente F, Molteni M, Marino B, et al. Bowel ultrasound and mucosal healing in ulcerative colitis. Dig Dis 2009;27:285-290. doi: $10.1159 / 000228562$

25. Novak KL, Kaplan GG, Panaccione R, et al. A Simple Ultrasound Score for the Accurate Detection of Inflammatory Activity in Crohn's Disease. Inflamm Bowel Dis 2017;23:2001-2010. doi:10.1097/ MIB.0000000000001174

26. Novak KL, Nylund K, Maaser C, et al. Expert consensus on Optimal Acquisition and development of the International Bowel Ultrasound Segmental Activity Score (IBUS-SAS): A reliability and inter-rater variability study on intestinal Ultrasonography in Crohn's Disease. J Crohns Colitis 2021;15:609-616. doi:10.1093/ecco-jcc/jjaa216

27. Bots S, Nylund K, Löwenberg M, Gecse K, D’Haens G. Intestinal ultrasound to assess disease activity in ulcerative colitis: development of a novel UC-Ultrasound Index. J Crohns Colitis 2021;15:1264-1271. doi:10.1093/ecco-jcc/jjab002

28. Saevik F, Eriksen R, Eide GE, Gilja OH, Nylund K. Development and validation of a simple ultrasound activity score for Crohn's Disease. J Crohns Colitis 2021;1:115-124. doi:10.1093/ecco-jcc/jjaa112

29. Medellin A, Merrill C, Wilson SR. Role of contrast-enhanced ultrasound in evaluation of the bowel. Abdom Radiol (NY) 2018;43:918-933. doi:10.1007/s00261-017-1399-6

30. Serafin Z, Białecki M, Białecka A, Sconfienza LM, Kłopocka M. Contrast-enhanced Ultrasound for Detection of Crohn's Disease Activity: Systematic Review and Meta-analysis. J Crohns Colitis 2016;10:354-362. doi:10.1093/ecco-jcc/jjv196 